\title{
Seat Occupancy and Belt Detection in Removable Seats via Inductive Coupling
}

\author{
Joan Albesa and Manel Gasulla \\ Department of Electronic Engineering, ISI Group \\ Universitat Politècnica de Catalunya (UPC) \\ Barcelona, Spain \\ joan.albesa@upc.edu
}

\begin{abstract}
Seat Belt Reminder (SBR) systems are effective in avoiding deaths and injuries in traffic accidents. However, their implementation can be unpractical in removable vehicle seats because of the difficulty in wiring the associated sensors, e.g. a seat occupancy sensor and a seat belt detector, to an electronic control unit (ECU). This paper proposes the use of inductive links to avoid wiring the sensors. Both sensors, which can be roughly modeled as switches, form part of a secondary resonant network. Their state (open- or short-circuit) is attained by estimating the resonance frequency of the equivalent input resistance of a primary network inductively coupled to the secondary network. Attending to the possible states of the sensors, four different resonant frequencies result. Because the application is space-constrained, small coils have been used. Commercial ferrite-core models were selected in order to achieve high coil inductance and quality factor. Furthermore, computer simulations showed the higher coupling factor achieved with respect to that achieved with air-core coils. Experimental tests were carried out using an impedance analyzer and commercial sensors for seat occupancy and belt detection. Detection was feasible at distances up to $2 \mathrm{~cm}$ between the primary and secondary coils.
\end{abstract}

Index Terms- Seat Belt Reminder, Inductive Link, Magnetic Coupling, Resonance Detection, Vehicles, Removable Seats.

\section{INTRODUCTION}

Passive safety systems in vehicles aim to reduce injuries of the occupants in an accident. Ref. [1] reports that the risk of fatal injuries is reduced by $45 \%$ in cars and $60 \%$ in vans just by using the seat belt. Despite the fact that wearing a seat belt is legally required in the EU, only few countries show driver rates of $90 \%$ and over. The non-users are not against using a seat-belt, they would use it if reminded by some device. A Seat Belt Reminder (SBR) system warns the driver that one or some of the occupants (including himself) have not buckled up the seat belt. The use of SBR systems is reported as one of the most effective ways in avoiding deaths and injuries in traffic accidents [2]. The Euro NCAP provides additional points to vehicles that incorporate SBR systems [3], thus facilitating the achievement of the maximum score (5 stars) for safety performance. SBR systems may also be essential for the proper control on the deployment of other passive safety devices such as air-bags. The costs for the implementation of SBR systems are small compared to the achieved benefits.

A SBR system for the driver seat consists of a seat belt detector wired to an electronic control unit (ECU). The passenger front-seat additionally includes an occupancy sensor in order to activate a warning only when the passenger is present and not buckled up.

Some vans and minivans incorporate removable seats in order to increase the flexibility in arranging their internal space. However, wiring this type of seats to incorporate the required sensors can be unpractical. A feasible option can be to use an inductive link. Some commercial models of vehicles incorporate this strategy for belt buckle detectors. A patent [4] proposes the detection of the state of switch sensors (open or closed), such as belt and occupancy detectors, in removable seats via an inductive link. However, neither analytical derivations nor practical implementations with experimental results are presented.

This paper proposes the use of inductive links for seat occupancy and belt detection in removable vehicle seats. The primary and secondary networks may be placed respectively in the car floor and at the bottom of the removable seat. In another paper, we propose to power remotely the secondary network instead [5].

Section II first shows the commercial sensors. Section III then presents the proposed detection technique and a theoretical analysis. Section IV presents the selected commercial coils and simulations of the coupling factor over their separation distance. Section V shows the measured performance and, finally, Section VI concludes the work.

\section{Seat OCCUPANCy SENSOR AND Belt Detector}

The commercial occupancy sensor (IEE company, Fig. 8) consists of a flexible sensor mat, which is inserted into the vehicle seat. The mat itself is composed of two sandwiched carrier sheets held together by an adhesive. Increased pressure on the sensor mat causes an electrical resistance change, which informs the system that the seat is occupied.

As a first approximation, the sensor can be modeled as an ideal switch. A vacant or occupied seat correspond respectively to an open- or short-circuit. Occupancy is detected for weights higher than $40 \mathrm{~kg}$. This permits to detect the presence of a passenger using a simple electronic interface. For diagnostic purposes, the manufacturer includes a resistor $(470 \Omega)$ in parallel with the sensor in order to differentiate the response of a vacant seat from the case in which any of the wires that connect the sensor with the ECU is broken. In this work, this parallel resistor was removed.

Joan Albesa enjoys a grant from the Ministry of Economy and Knowledge of the Regional Government of Catalonia, Spain, and the European Social Funding on the FI program. He also enjoyed a Mobility grant from the Ministry of Education of Spain and an UPC-Enterprise grant from the car company SEAT and the Technical University of Catalonia (UPC). 
The seat belt detector (TRW Sabelt company) consists of a buckle and the corresponding buckle housing, which can also be modeled as an ideal switch. An unbuckled or buckled up seat belt can be respectively modeled as a short- or opencircuit.

\section{DETECTION TECHNIQUE}

Inductive links have been widely proposed to sense capacitive sensors in harsh or inaccessible environments, where no wiring between the sensor and the processing unit is practicable [6], [7]. In these systems, the sensor is disposed together with an inductor (secondary coil) forming a resonant circuit (secondary network), whose resonant frequency changes according to the value of the capacitive sensor. A readout unit (primary network), which incorporates another inductor (primary coil), is magnetically coupled to the sensor unit.

A related technique was proposed in a patent [4] for switch sensors in vehicles, although no theoretical analysis and experimental results were provided. Here, we further develop this technique for seat occupancy and belt detection. Fig. 1 illustrates the circuit model for the sensors and the inductive link. $L_{1}$ and $L_{2}$ are the primary and secondary coils, $R_{1}$ and $R_{2}$ model their respective losses, and $M$ is the mutual inductance. The seat occupancy sensor and the belt detector are modeled by switches $S_{1}$ and $S_{2}$, respectively. $C_{2}, C_{\mathrm{S} 1}$ and $C_{\mathrm{S} 2}$ are discrete capacitors of appropriate value.

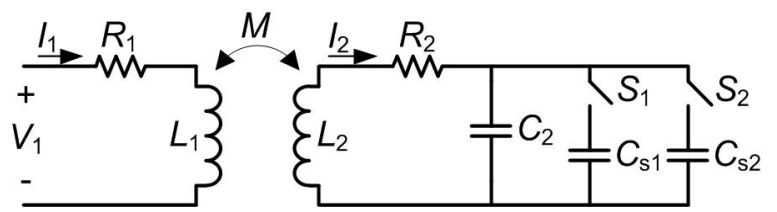

Figure 1. Circuit model for the seat occupancy and belt detector and the inductive link.

In [7], a review of different readout circuit architectures is presented. The authors advocate for a technique based on the measurement of the real part of the impedance measured from the primary coil. By detecting its resonance frequency, the value of the capacitance can be obtained. Here, we adopt this technique.

The impedance seen from the primary coil is given, using complex notation, by [7]

$Z_{1}=\frac{V_{1}}{R_{1}}=R_{1}+j \omega L_{1}\left(1+\frac{k^{2}\left(\frac{\omega}{\omega_{\mathrm{r}}}\right)^{2}}{1+j \frac{1}{Q_{\mathrm{T}}} \frac{\omega}{\omega_{\mathrm{r}}}-\left(\frac{\omega}{\omega_{\mathrm{r}}}\right)^{2}}\right)$

where $k$ is the coupling factor between the coils $\left(M=k \sqrt{L_{1} L_{2}}\right)$,

$\omega_{\mathrm{r}}=\frac{1}{\sqrt{L_{2} C_{\mathrm{T}}}}$

is the resonance frequency, and

$Q_{\mathrm{T}}=\frac{1}{R_{2}} \sqrt{\frac{L_{2}}{C_{\mathrm{T}}}}$
$C_{\mathrm{T}}=C_{2}+S_{1} C_{\mathrm{s} 1}+S_{2} C_{\mathrm{s} 2}$

where $S_{1}$ and $S_{2}$ in Fig. 1 equal to 0 or 1 whenever they correspond to an open- or short-circuit. Hence, four different cases result:

1) $S_{1}=0, S_{2}=1: C_{\mathrm{T}}=C_{2}+C_{\mathrm{s} 2}$; vacant seat, unbuckled.

2) $S_{1}=1, S_{2}=1: C_{\mathrm{T}}=C_{2}+C_{\mathrm{s} 1}+C_{\mathrm{s} 2}$; occupied seat, unbuckled .

3) $S_{1}=0, S_{2}=0: C_{\mathrm{T}}=C_{2}$; vacant seat, buckled up.

4) $S_{1}=1, S_{2}=0: C_{\mathrm{T}}=C_{2}+C_{\mathrm{s} 1}$; occupied seat, buckled up.

The SBR system must warn in case 2 .

From (1), the real part of $Z_{1}$ is given by

$$
\operatorname{Re}\left\{Z_{1}\right\}=R_{1}+\omega L_{1} k^{2} Q_{\mathrm{T}} \frac{\omega / \omega_{\mathrm{r}}}{1+Q_{\mathrm{T}}^{2}\left(\frac{\omega}{\omega_{\mathrm{r}}}-\frac{\omega_{\mathrm{r}}}{\omega}\right)^{2}}
$$

which presents a maximum at

$$
\omega_{\max }=\omega_{\mathrm{r}} \frac{1}{\sqrt{1-\frac{1}{2 Q_{\mathrm{T}}^{2}}}} .
$$

Whenever $Q_{\mathrm{T}}>1$, thus $\omega_{\max } \approx \omega_{\mathrm{r}}$ and

$$
\left.\operatorname{Re}\left\{Z_{1}\right\}\right|_{\omega=\omega_{\mathrm{r}}}=R_{1}+k^{2} \omega_{\mathrm{r}} L_{1} Q_{\mathrm{T}} \text {. }
$$

By searching the maximum value of $\operatorname{Re}\left\{Z_{1}\right\}$ we will obtain $\omega_{\mathrm{r}}$. Then, from (2), the value of $C_{\mathrm{T}}$ can be inferred and thus the state of $S_{1}$ and $S_{2}$.

\section{COILS AND COUPLING FACTOR}

A rather low frequency of operation $(<150 \mathrm{kHz})$ was sought for two reasons: 1) to comply with the reference levels for general public exposure to time-varying electric and magnetic fields [8], and 2) to ease the design of the electronic circuitry for the measurement of the real part of the impedance. Furthermore, for the intended application, small-size coils are a requirement. So, in order to increase $k, L_{1}$, and $Q_{\mathrm{T}}$ in (7), the use of magnetic-core material for the coils was considered as an appropriate solution.

We selected $1 \mathrm{mH}$ commercial coils from Fastron (PIST model) which present a dc resistance of $1.5 \Omega$. Fig. 2 shows the dimensions of the coils (values are in millimeters).

is the quality factor of the coils. $C_{\mathrm{T}}$ is given by 

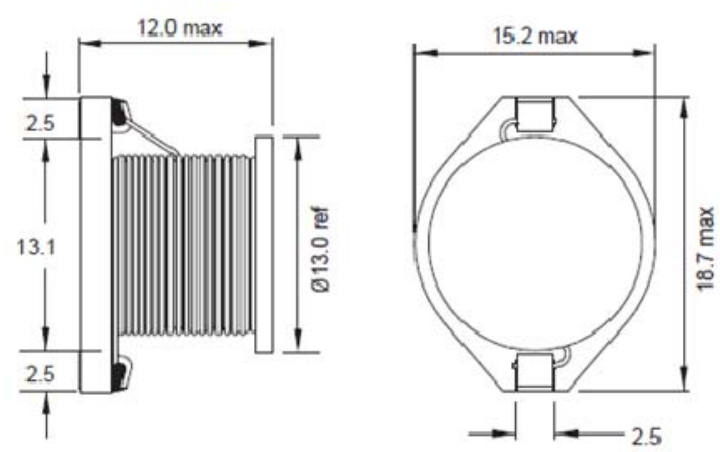

Figure 2. Dimensions of the selected $1 \mathrm{mH}$ coils(in millimeters). Source: http://www.fastrongroup.com/.

In order to obtain the experimental quality factor of the coil at different frequencies, we used a series-resonant network and measured the resistance at the resonant frequency by using an HP4194A impedance analyzer. Appropriate values of capacitors were used in order to tune the resonant frequency. Fig. 3 shows the measured values of the quality factor. The quality factor increased up to a maximum (ca. 40) at $40 \mathrm{kHz}$ and then decreased for higher values of frequency. This is due to the increase of coil losses with frequency because of the joint combination of skin and proximity effects and the losses of the ferrite.

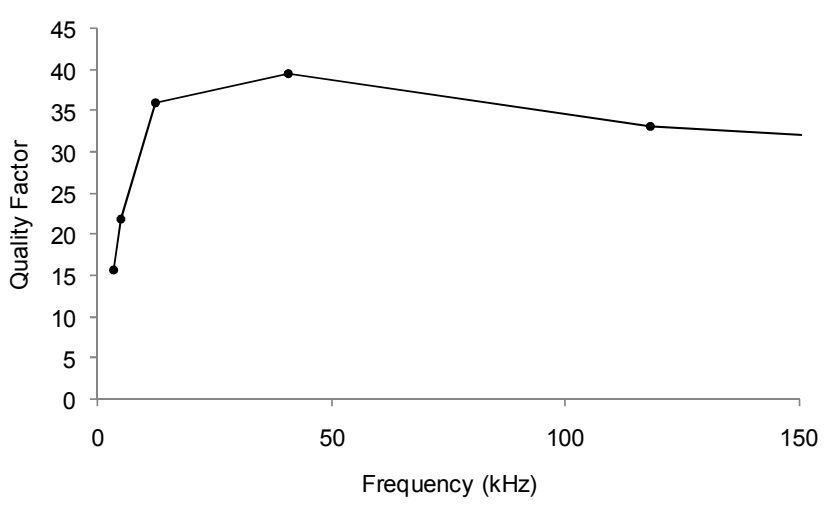

Figure 3. Measured values of the coil quality factor.

In order to estimate the self and mutual inductance of the coils, and the coupling factor between the coils over their separation distance $(d)$, we used the simulation program COMSOL. Fig. 4 shows an axisymetric model for the primary and secondary coils, where $d$ is marked. Sizes of the coils were in accordance with those presented in Fig. 2. Both, ferrite- and air-core coils were used in order to compare their performance. The contour areas R1 to R6 were defined either as ferrite or air whereas C1 and C2 (wire coil) were defined as copper. A relative permittivity $\left(\mu_{\mathrm{r}}\right)$ of 2000 was used for the ferrite. Spherical domain boundaries were used and set to zero magnetic insulation.

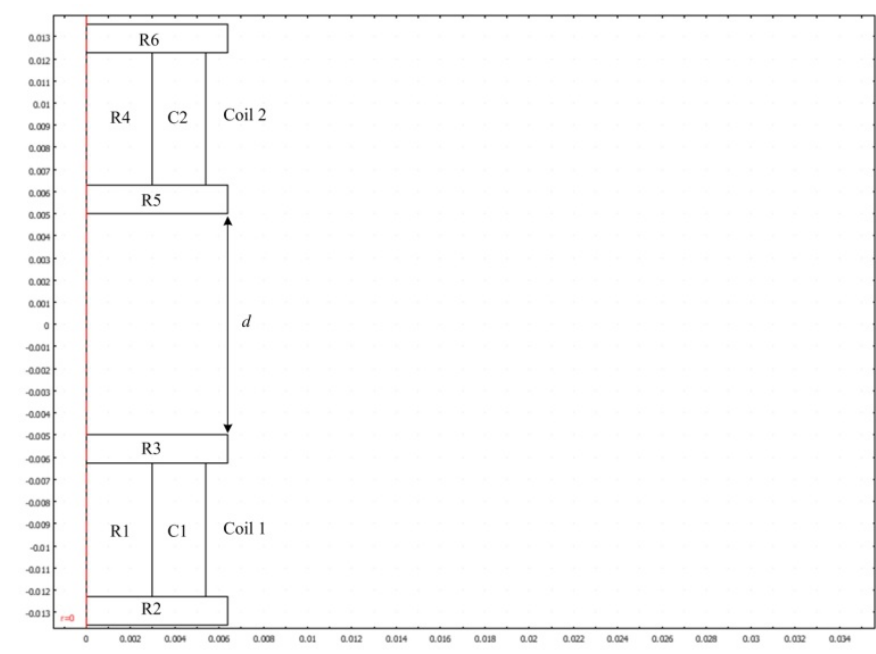

Figure 4. Modeling of the primary and secondary coils using COMSOL.

The self inductance of the coils was calculated in DC (no remarkable differences with $\mathrm{AC}$ simulations for frequencies lower than $150 \mathrm{kHz}$ were found) according to

$$
L=\frac{N^{2} \bar{\phi}}{\iint_{S} J_{\varphi}^{e} d r d z}
$$

where $N$ corresponds to the number of turns, $\bar{\phi}$ is the average of the magnetic flux, and $J_{\varphi}^{\mathrm{e}}$, is the current density applied over the conductive surface of the considered coil. The mutual inductance $M$ is calculated in the same way but now the magnetic flux is generated by the current density of the other coil. Finally $k$ is calculated as

$$
k=\frac{M}{L} \text {. }
$$

For the simulations we used $N=130$. The value of $L$ was about ten times larger for ferrite-core coils (about $1 \mathrm{mH}$ ) than for air-core coils (about $100 \mu \mathrm{H}$ ). For ferrite-core coils, the value of $L$ noticeably increased for very short distances due to the presence of the ferrite of the other coil. This effect can be appreciated in Fig. $5(d=0.5 \mathrm{~cm})$, where the magnetic field lines generated by the bottom coil were distorted by the presence of the ferrite of the top coil, leading to an increase of $L$. This effect is not present in air-core coils (Fig. 6). As can also be seen, with ferrite-core coils, more magnetic field lines of the bottom coil go through the top coil, thus increasing $k$. Fig. 7 shows graphically the evolution of $\mathrm{k}$ for different distances, from $0 \mathrm{~cm}$ to $3 \mathrm{~cm}$ in steps of $0.5 \mathrm{~cm}$. The value of $k$ was about 3 to 4 times larger for ferrite- than for air-core coils. 


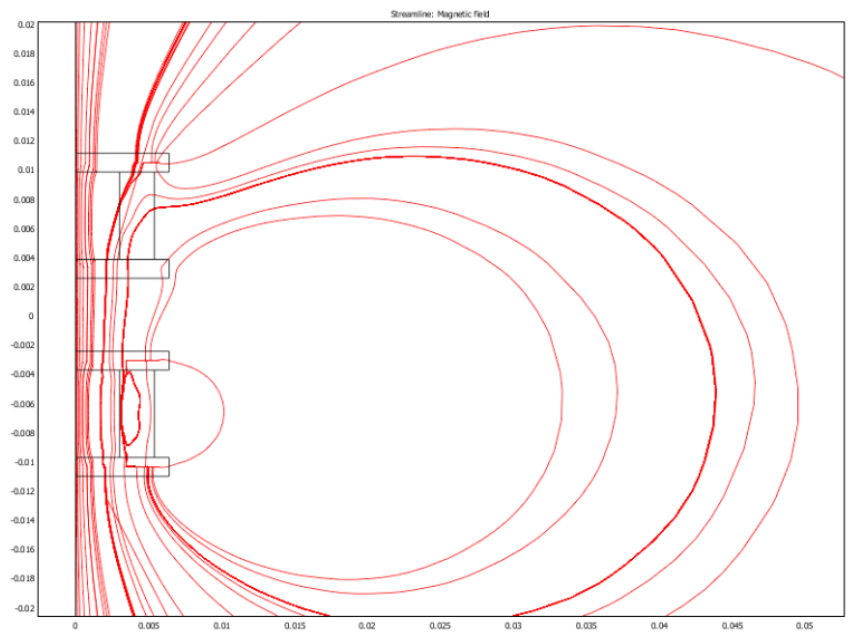

Figure 5. Captured images in COMSOL of the magnetic field lines of two ferrite-core coils separated $0.5 \mathrm{~cm}$. The current density was applied on the bottom coil.

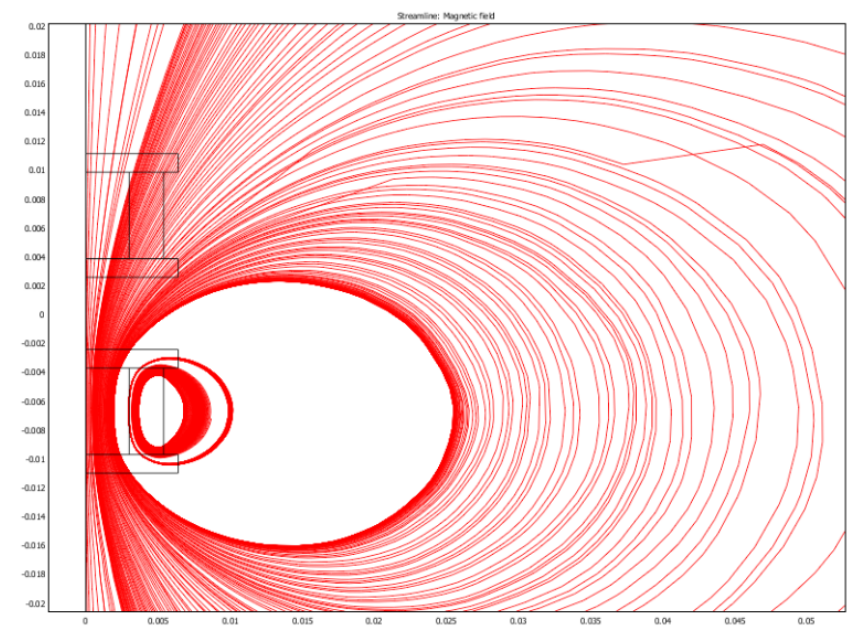

Figure 6. Captured images in COMSOL of the magnetic field lines of two air-core coils separated $0.5 \mathrm{~cm}$. The current density was applied on the bottom coil.

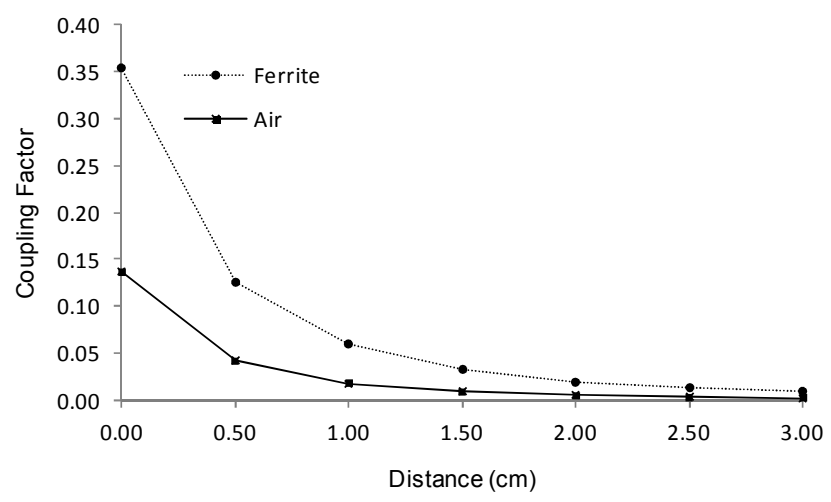

Figure 7. Evolution of $k$ over $d$ for ferrite- and air-core coils.

\section{PERFORMANCE}

Fig. 8 shows the mechanical setup fabricated to fix the distance between the primary and the secondary networks, which were implemented in separate PCB boards. Distance was adjusted manually. The support and the fixing screws were made of nylon. The picture also shows the occupancy sensor and the car seat that incorporates it. The primary network was connected to an impedance analyzer (HP4194A) in order to measure the real part of the impedance. In reference to Fig. 1, we use the $1 \mathrm{mH}$ commercial coils, $C_{2}=C_{\mathrm{s} 1}=10 \mathrm{nF}$ and $C_{\mathrm{s} 2}=22 \mathrm{nF}$ and the commercial sensors for seat occupancy and belt detection. Thus, the resulting nominal resonant frequencies corresponding to the four possible states of the sensors (see section III) were: $50.4 \mathrm{kHz}, 35.6 \mathrm{kHz}, 28.2 \mathrm{kHz}$, and $24.6 \mathrm{kHz}$.

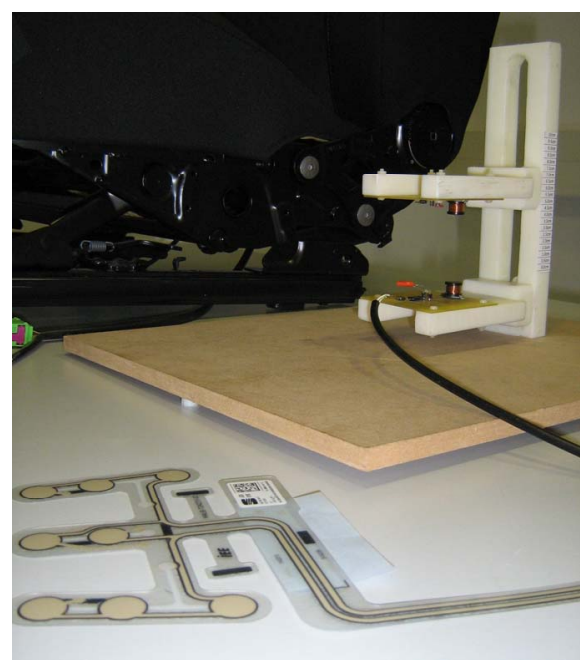

Figure 8. Mechanical setup used to fix the distance of the primary and secondary networks. The occupancy sensor is also shown.

Fig. 9 shows the measured values of $\operatorname{Re}\left\{Z_{1}\right\}$ for the case of an occupied seat and unbuckled belt $\left(S_{1}=1, S_{2}=1\right)$ at four different distances $(0.5 \mathrm{~cm}, 1 \mathrm{~cm}, 1.5 \mathrm{~cm}$, and $2 \mathrm{~cm}$, in colors). The SBR system must warn the driver in this case. As can be seen, the maximum value of $\operatorname{Re}\left\{Z_{1}\right\}$ increased for decreasing distances. From Fig. 7, a decrease of distance leads to an increase of $k$ and thus, from (7), to an increase of the maximum value of $\operatorname{Re}\left\{Z_{1}\right\}$. Furthermore, the resonant frequency slightly decreased for shorter distances. This is due to the increase of the self-inductance, as mentioned in section IV. 


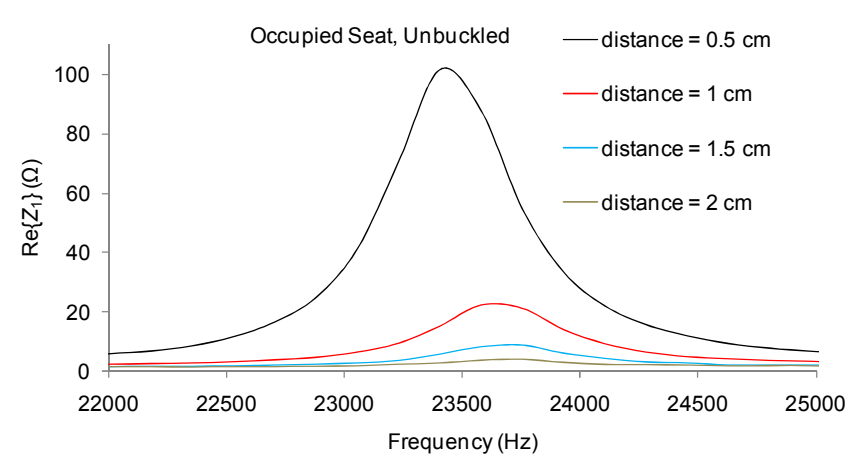

Figure 9. Real part of the impedance for the case of an occupied seat and unbuckled belt at different distances. The SBR system must warn the driver in this case.

Fig. 10 shows the measured values of $\operatorname{Re}\left\{Z_{1}\right\}$ for the four states of the sensors and at the four referred distances in Fig. 9. As can be seen, the respective resonant frequencies can be clearly distinguished, which allows to determine the state of both sensors. A lower quality factor $\left(Q_{\mathrm{T}}\right)$ can be observed for the cases where the seat is occupied. This is due to the finite resistance presented by the occupancy sensor ( $15 \Omega$ measured).

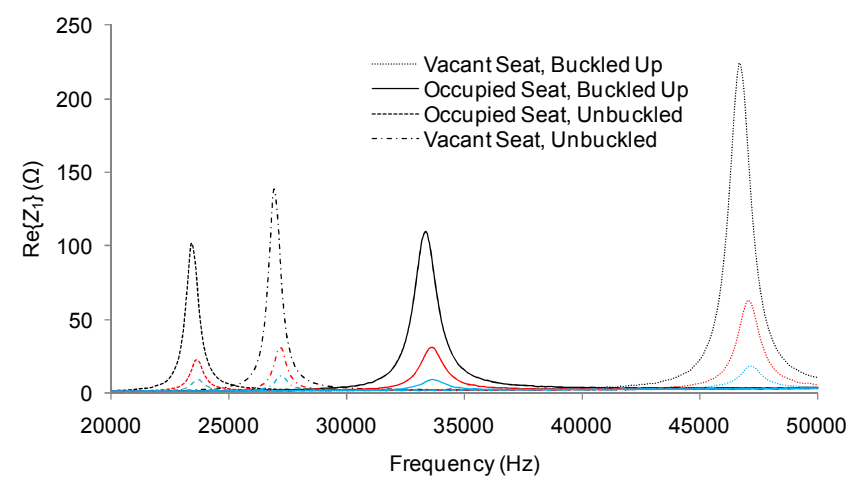

Figure $10 . \operatorname{Re}\left\{Z_{1}\right\}$ for the four different states of the sensors.

\section{CONCLUSIONS}

We have proposed and tested a technique for seat occupancy and belt detection in removable vehicle seats via an inductive link. This avoids physically wiring the sensors to an ECU, which can become unpractical.

Both sensors, which are simply modeled as switches, form part of a secondary resonant network. The state of the sensors is attained via the resonance frequency of the real part of the impedance of a primary network inductively coupled to the secondary network. Attending to the sensors' states, four different resonant frequencies result.

Because the application is space-constrained, small coils have to be used. Commercial ferrite-core models have been selected in order to achieve high coil inductance and quality factor, as well as a high coupling factor between the primary and secondary coils. Simulations have shown the benefits of using this approach.

Experimental tests have been carried out using an impedance analyzer and a commercial sensors for seat occupancy and belt detection. A mechanical setup has been fabricated to fix the distance between the primary and secondary networks. Results have been obtained at four distances, from $0.5 \mathrm{~cm}$ to $2 \mathrm{~cm}$, in $0.5 \mathrm{~cm}$ steps. For each distance, four resonant frequencies can be clearly distinguished corresponding to the four possible states of the sensors. Resulting quality factors are higher for shorter distances, facilitating the detection.

\section{ACKNOWLEDGMENT}

The authors acknowledge the support of the car company SEAT in providing the occupancy sensor and the car seat.

\section{REFERENCES}

[1] A. Lie, A. Kullgren, M. Krafft, and C. Tingvall C, "Intelligent Seat Belt Reminders: Do they Change Driver Seat Belt use in Europe?," The 20th International Technical Conference on the Enhanced Safety of Vehicles (ESV), Paper number: 07-0388, 2007.

[2] ETSC, "Seat Belt Reminders. Implementing advanced Safety Technology in Europe's Cars," 2006.

[3] Euroncap, "Seat Belt Reminder Assessment Protocol. Version 1.2," 2007.

[4] R. Darraba, "A Movable or Removable Seat for a Motor Vehicle," WO 2004/098943 A1, Nov 18, 2004.

[5] J. Albesa, T. Jäger, L. M. Reindl, and M. Gasulla, "Wireless Power Transmission for Autonomous Sensors in Removable Vehicle Seats", IEEE VTC2011-Fall (accepted).

[6] J. Garcia-Canton; A. Merlos, and A. Baldi, "A wireless LC chemical sensor based on a high quality factor EIS capacitor," Sensors and Actuators B, vol.126, pp.648-654, 2007.

[7] R. Nopper, R. Niekrawietz, and L. Reindl, "Wireless Readout of Passive LC Sensors," IEEE Transactions on Instrumentation and Measurement, vol.59, no.9, pp.2450-2457, Sept. 2010.

[8] International Commission on Non-Ionizing Radiation Protection, [ICNIRP] "Guidelines for limiting exposure to time-varying electric, magnetic, and electromagnetic fields," Health Physics, vol.74, no.4, April 1998. 